\title{
COMENTARIOS AL CÓDIGO PROCESAL CIVIL. LA POSTULACIÓN DEL PROCESO
}

\author{
Carlos Parodi Remón
}

El tema que hemos escogido para este comentario es uno de los que ofrece mayores modificaciones con respecto a la legislación anterior. En el Código de Procedimientos Civiles los requisitos de la demanda contenidos en su artículo 306 eran solamente cuatro, en tanto que el artículo 424 del nuevo Código incluye once; pero no se trata solamente del número de requisitos, es decir de su cuantificación, sino de aspectos mucho más importantes que pueden incidir en el fondo mismo del asunto, lo que amerita un análisis más profundo del problema, pues en opinión nuestra, las cuestiones relativas a la postulación, a la posibilidad de demandar para obtener una sentencia justa, al derecho de requerir un pronunciamiento jurisdiccional, están ligadas con algunas premisas previas, como el acceso a la justicia y la necesidad, especialmente en nuestro medio, de acercar el justiciable al juez, el Derecho al Hombre, que son, también, sin duda, principios inspiradores del Código Procesal Civil. Reflexionemos pues al respecto, incidiendo en los artículos 426 y 427 del mismo.

El artículo 426 autoriza al juez a declarar inadmisible la demanda cuando no tenga los requisitos legales, no se acompañen los anexos exigidos por la ley, el petitorio sea incompleto o impreciso o la vía procedimental propuesta no corresponda a la naturaleza del petitorio o al valor de éste, salvo que la ley permita su adaptación. En estos casos el juez otorga al demandante un plazo de 10 días para que subsane la omisión o defecto y, de no cumplir, el juez rechaza la demanda y ordena archivar el expediente.

Esta norma deviene una versión ampliada y obviamente mejorada de la contenida en el artículo 307 del Código de Procedimientos Civiles, pero 
se entiende que el «archivo del expediente» al que se refiere el último párrafo del artículo 426, no impide al demandante frustrado reiniciar la demanda al cumplir con todos los requisitos legales. Tal conclusión deriva de la naturaleza de los requisitos que dicha norma contiene, formales todos ellos, y porque parece injusto o exagerado que la falta de un requisito formal que, desde un punto de vista genérico, puede enmendarse en cualquier momento, elimine para siempre la posibilidad del pedido del reconocimiento de un derecho. Coincidimos con la necesidad de aligerar el trámite de los procesos y de sancionar la presentación de demandas de mala fe o carentes de los elementales requisitos, pero no a costa de privar a cualquier persona del derecho que tiene a acudir a la vía jurisdiccional. En la práctica esta posibilidad puede convertirse en una traba al acceso a la justicia, algo inaceptable, y esperamos que la jurisprudencia así lo establezca.

Abunda en favor de tal solución el término «incompleto» que encontramos en el inciso 3 del artículo 426, pues tal supuesto depende en todo caso del mismo demandante y no del juez, quien debe limitarse, en este aspecto, a ponderar la demanda, pero no a decidir sobre los extremos de su contenido. En otros términos, que la demanda sea completa o incompleta es un aspecto propio del demandante, que el juez evaluará al expedir sentencia, aplicando el principio de congruencia.

Tampoco se compadece con la rotundidad de la conclusión, de «archivar el expediente», la regla del inciso 4 del mismo artículo 426 , pues si la vía procedimental propuesta «no corresponde a la naturaleza del petitorio», el juez puede hacer uso de la facultad que le conceden los artículos 475 inciso $1,477,486$ inciso 8 y 546 inciso 6 , los cuales, en una interpretación correcta acorde con los principios de celeridad y economía procesales que informan el contenido del Código, confieren al juez la facultad de adaptar la vía procedimental a la naturaleza del petitorio, como lo dice expresamente el artículo 477, solución con la cual coincidimos y que se inscribe, además, en la figura del juez «director del proceso», expresado, entre otros, en el artículo II del Título Preliminar y artículo 50 inciso 1, y en especial en el artículo 51 inciso 1, cuya claridad nos exime de mayor comentario y el cual debe tenerse en cuenta por el juez al aplicar el artículo 426 inciso 4, el mismo que debe ser considerado, en todo caso, restrictiva y no extensivamente.

Tal solución, que proponemos, se inscribe dentro del marco debido del «acceso a la justicia». 
Lo que venimos manifestando cobra aun mayor dimensión al analizar el artículo 427 en sus dos primeros incisos, relativos a la falta de legitimidad para obrar y a la falta de interés, casos en los cuales el juez puede declarar la demanda improcedente, lo que eventualmente podría resultar riesgoso.

Este riesgo proviene en primer lugar de la diferencia entre la «inadmisibilidad» y la «improcedencia». La primera se refiere a requisitos de forma y, como hemos visto, no impide que en su ausencia o defecto el interesado pueda reformular su demanda. En cambio la segunda incide en aspectos más profundos, de fondo si se quiere; y es por ello que, según el último párrafo del artículo 427, la resolución que declara la improcedencia es apelable con efecto suspensivo, resolviéndose en definitiva la improcedencia con efectos para ambas partes. Tal solución, acorde en principio con los supuestos del artículo 427, acredita pues un análisis de la situación.

En nuestra opinión son los dos primeros incisos, los referidos a la legitimación para obrar y al interés, los que pueden producir mayores problemas, en especial en un medio como el nuestro, en el que el acceso a la justicia se encuentra limitado a un determinado sector de la población por razones socioeconómicas que no es del caso reseñar.

Estimamos que la «legitimación para obrar» y el «interés» son propios de la evalución que el juez deberá realizar al expedir sentencia y no es conveniente que puedan ser ponderados «a priori», como se desprende del tenor del comentado artículo 427.

En efecto, ¿qué es la legitimación en causa o legitimidad para obrar? «Entendemos como legitimación en causa la condición o cualidad de carácter procesal que el ordenamiento legal sustantivo reconoce a una determinada categoría de sujetos (acreedores, herederos, accionistas, contratantes, etc.) que faculta a éstos para pretender sobre una concreta relación jurídica, en el caso del demandante, y en el del demandado, para oponerse a las pretensiones esgrimidas en su contra». «Es la condición que debe tener una persona según la ley sustantiva para lograr que el juez se pronuncie respecto a las pretensiones formuladas en la demanda en relación con una concreta y par- 
ticular relación jurídica» ${ }^{1}$. Tales conceptos, emitidos por el eminente procesalista panameño Jorge Fábrega, nos llevan de la mano para arribar a la solución más idónea en este aspecto del artículo 427. El mismo jurista nos señala los caracteres que diferencian la legitimación para obrar de otras instituciones, expresando que: «La legitimación en la causa no es una condición para ejercer el derecho de acción». «La legitimación en la causa no es lo mismo que la titularidad del derecho material». «La legitimación en la causa es una noción distinta de la capacidad para ser parte». «La legitimación en la causa es el extremo opuesto de las denominadas acciones populares». «La legitimación en la causa no es suficiente para obtener sentencia favorable». "La legitimación en la causa es materia que, en términos generales, debe ser dilucidada en la sentencia que decide el proceso». "La falta de legitimación en la causa puede ser declarada de oficio». «La legitimación en la causa no es susceptible de cesión»².

Si bien es cierto que el tratadista mencionado al comentar la antepenúltima afirmación agrega que pueden darse algunas excepciones, en las cuales «la ley exige que desde el comienzo el juzgador examine el punto de si se tiene o no legitimación en la causa», como en los casos de la tercería excluyente, el tercero que actúa como litisconsorte, o el interdicto de adquirir (figura eliminada en el nuevo Código), ellos son supuestos específicos y no decisivos para sostener que la legitimación en la causa es un aspecto que debe decidirse en la sentencia.

En el mismo sentido se pronuncia el procesalista español Francisco Ramos Méndez, al afirmar: «En mi opinión, la teoría de la legitimación representa hoy un esfuerzo superfluo y vano para tratar de asegurar la presencia en el proceso, desde su iniciación, del verdadero titular del derecho discutido en el juicio. Dicho propósito es estéril porque, como veremos, admitido el principio de libertad de acción, no puede condicionarse la admisibilidad del proceso a que el litigante justifique in limine litis la titularidad de su derecho. La existencia o no de dicha titularidad sólo resultará del juicio, de la sentencia, en definitiva. Lo que bastará para la conduccion del proceso es sencillamente que dicha persona sea una de los

1 Fábrega, P. Jorge, Estudios procesales, tomo I, Editora Jurídica Panameña, Panamá, 1989, págs. 247-251.

2 Fábrega, ob. cit., págs. 248-255. 
litigantes». Agrega: «El hecho de que una persona sea o no propietaria o acreedora es algo que sólo resulta de la sentencia que pone término al proceso, pero que no se sabe inicialmente». «Están legitimadas en un proceso concreto las partes procesales, por el mero hecho de serlo. Quiénes son estas partes procesales nos lo dice la demanda. Por lo tanto, estará legitimada activamente en un proceso concreto la persona que proponga la demanda y legitimada pasivamente la persona frente a quien se dirige la demanda. La introducción de un proceso se hace en base a una afirmación jurídica que efectúa una de las partes. Ello, por sí solo es suficiente para fundar la legitimación de las partes en ese proceso y generar todo el conjunto de expectativas y cargas en que éste se resuelve. Al inicio del proceso, atribuir otro significado distinto a la legitimación es basarse en una imagen pedagógica de lo que en su día puede resultar de la sentencia» ${ }^{3}$.

No está de más recordar que el problema comentado se refiere a la legitimación para obrar, esto es, la «legitimatio ad causam», diferente obviamente a la «legitimatio ad processum» referida a la capacidad procesal y respecto de la cual parte de la doctrina estima conveniente su no tratamiento para evitar confusiones, tanto de léxico cuanto de contenido.

Similar situación se presenta con el inciso 2 del artículo 427 del Código Procesal Civil, según el cual también el juez declara la demanda improcedente cuando «el demandante carezca manifiestamente de interés para obrar». Al respecto dice Fábrega: «Otra de las nociones que cobra especial importancia al momento de dictar fallo de fondo, es el atinente al examen del interés sustancial que deben tener las partes que actúan en el proceso", definiéndolo como «el motivo o razón de carácter jurídico material, serio y particular que lleva a una persona (en el caso del demandante) a procurar la intervención de los órganos jurisdiccionales del Estado a fin de que se acceda a las pretensiones formuladas en la demanda; y en el caso del demandado, la razón por la cual se opone o contradice tales pretensiones. Ese interés también alcanza a los terceros que intervienen en el proceso en cualquiera de las modalidades permitidas en la ley (coadyuvancia, ad excludendum, etc.)». Al igual que en el caso de la legitimación para obrar, el jurista panameño distingue al interés para obrar de otras nociones, expresando que: «El

3 Ramos Méndez, Francisco, Derecho procesal civil, Librería Bosch Barcelona, España, 1980, págs. 240-244. 
interés sustancial para obrar es distinto del interés para ejercitar el derecho de acción». «El interés sustancial para obrar no es lo mismo que la titularidad del derecho material pretendido», agregando que los criterios para identificarlos son:

«Al momento de decidir el proceso debe examinarse si las partes tienen un interés sustancial concreto, esto es, referido a una particular y específica relación o situación jurídica.

Debe analizarse si con la promoción de la demanda o con la oposición a ella, la parte obtiene un beneficio o un perjuicio, el cual puede ser patrimonial, moral, social, etc.

Debe examinarse, por último, que el interés sea actual, es decir, que se apoye en hechos ya acaecidos, pues las declaraciones jurisdiccionales no pueden estar supeditadas a hechos inciertos».

Finalmente, el tratadista centroamericano nos dice que: «El efecto que se produce cuando falta en alguna de las partes el interés sustancial para obrar es la dictación de una sentencia desfavorable», afirmando que para que «el juez pueda dictar sentencia favorable a las pretensiones indicadas en la demanda, es necesario que concurran tres condiciones básicas: a) Estar legitimado en la causa; b) Tener la titularidad del derecho material, c) Gozar de un interés sustancial para obrar» ${ }^{4}$. (Todos los subrayados son nuestros).

Ramos Méndez coincide con la opinión expuesta en el sentido de que el interés debe estar referido a la sentencia y no a la demanda, al decir: «En general no puede negarse que todo litigante, por el mero hecho de serlo, tiene interés en el ejercicio de su acción en el proceso. Eso es obvio, pues el interés en obrar se traduce en el incoamiento del proceso y en la actividad procesal», reflexionando luego: «Así como en las acciones llamadas gráficamente constitutivas o de condena parece que no hay inconveniente alguno en admitir la existencia de un interés genérico por el mero hecho de incoar el proceso y de lo que se pide en el mismo, no ocurre otro tanto en el sentir de la jurisprudencia, respecto de las llamadas acciones meramente declarativas. En éstas se viene exigiendo la concurrencia de un interés que

${ }^{4}$ FábreGA, ob. cit., págs. 262-266. 
condicione la intervención jurisdiccional como única medida posible para la satisfacción de dicho interés. A mi entender, con ello se está refiriendo una vez más la jurisprudencia al tema de fondo, a la decisión que se debe adoptar en su día, lo cual no puede condicionar la admisibilidad del proceso» ${ }^{5}$. (El subrayado también es nuestro).

No cabe pues duda sobre el pensamiento de ambos ilustres autores sobre la legitimación para obrar y el interés, ambos elementos determinantes, no de la presentación y admisión de una demanda, sino de la expedición de una sentencia favorable a quien pidió el reconocimiento o la declaración de un derecho. Condicionan ésta, mas no la presentación de una demanda, a la que se puede considerar como «la expresión material de la acción que afirma y sostiene una pretensión».

El análisis que estamos proponiendo en manera alguna puede considerarse meramente teorico. Tal como advertimos antes, el tratamiento de estos conceptos está ligado muy de cerca al tema general del «acceso a la justicia», tan en auge en la doctrina actual y, a su vez, inherente a un marco más extensivo como es el contorno socioeconómico referido al mundo en que vivimos. Es, decíamos, preocupación constante de la doctrina jurídica, en especial la procesal, el establecer claramente el derecho absolutamente incondicionado de la persona, cualquiera que sea ella, a acceder a la justicia, tema que ha sido y es materia de numerosos trabajos, entre los que destaca el dirigido y elaborado por el ilustre procesalista italiano Mauro Cappelletti, al que glosa Roberto Berizonce como «una colosal investigación conocida como el Proyecto Florentino sobre el acceso a la justicia» ${ }^{6}$. Este punto tiene que ver directamente con el postulado primordial de toda reforma que aspira a serlo: la necesidad de acercar el ser humano al servicio de justicia como premisa ineludible para alcanzar la paz social. La legislación así lo ha comprendido como en los dos primeros párrafos del artículo 7 de la Constitución mexicana, el punto 1 del artículo 24 de la Constitución española, el artículo 11.1 del proyecto del Código Procesal Civil Modelo para Iberoamérica, entre otros, y entre nosotros, el segundo párrafo del artículo 7

5 Ramos, ob. cit., págs. 244-245.

- Berizonce, Roberto O., en la «Presentación» al libro El acceso a la justicia de Mauro Cappelletti y Bryant Garth, Colegio de Abogados del Departamento Judicial de La Plata, Argentina, 1983. 
de la Ley Orgánica del Poder Judicial, así como el Código Procesal Civil en el artículo I de su Título Preliminar y en el artículo 2 de su Título I, pero ninguna norma más clara que la contenida en el artículo 32 de la Constitución Imperial del Japón del 3 de noviembre de 1946: «A nadie se le podrá negar el derecho de acceso a los tribunales de justicia».

Sin embargo, para que el «acceso a la justicia» sea una posibilidad viable y no una mera declaración teórica, se requiere determinadas premisas concretas, entre ellas, la primera tal vez, el ejercicio absolutamente incondicionado del derecho de acción, tal como lo dispone claramente el artículo 3 del mismo Código Procesal Civil, debiendo entenderse que los «requisitos procesales» a que dicha norma se refiere, inciden en la demanda misma, pero no en el ejercicio de la acción. De otro modo sería limitar ésta, lo que no puede admitirse. Obviamente que en determinados casos la persona no puede ejercer ella misma la acción, como en los supuestos de los menores de edad, mayores incapaces, etc., pero ello no significa que el ejercer la acción padezca alguna limitación y además se trataría, en todo caso, de problemas relacionados con la «capacidad para ser parte» o la «capacidad procesal», pero siempre manteniendo incólume el derecho de acción, por lo demás previsto en los artículos 8 y 10 de la Declaración Universal de los Derechos Humanos, artículo 8 de la Convención Americana sobre Derechos Humanos, artículo XVIII de la Declaración Americana de Derechos y Deberes del Hombre y artículo 14 del Pacto Internacional de Derechos Civiles y Políticos.

Es contundente la opinión de Couture sobre la acción: «Este poder jurídico compete al individuo en cuanto tal, como un atributo de su personalidad». «Entendemos pues, por acción, no ya al derecho material del actor ni su pretension a que ese derecho sea tutelado por la jurisdicción, sino su poder jurídico de acudir ante los órganos jurisdiccionales»?

La acción, pues, es un derecho humano. Y no obstante la excelsitud del nivel que dicho concepto ha alcanzado, la doctrina resulta ilimitada y permanente en su evolución. Así, Fix Zamudio, al comentar las instituciones procesales fundamentales, como la acción, la jurisdicción y el proceso,

7 Couture, Eduardo, Fundamentos del Derecho procesal civil, Depalma, Buenos Aires, 1958, págs. 57-61. 
expresa que «no se trata de un simple acceso a la prestación jurisdiccional, sino que se traduce en el derecho fundamental a la justicia» ${ }^{8}$.

Así las cosas, no cabe duda de que el acceso a la justicia sólo se consigue con el ejercicio del derecho de acción, pero como ésta, derecho humano, no se agota ni mucho menos con la simple presentación de la demanda, sino que subyace en todo el proceso hasta su culminación, que incluye no sólo la sentencia misma, sino la cosa juzgada que ésta produce y la reivindicación de la paz social alterada por el conflicto de intereses, resulta clara la necesidad de viabilizar la posibilidad de cualquier persona para acudir a los tribunales en demanda de la declaración o el reconocimiento de un derecho, lo que también comprende, obviamente, el derecho de la persona demandada a ejercer ese mismo derecho en vía de defensa o de contradicción.

El problema pues se contrae a armonizar lo mejor posible la incondicionalidad en el ejercicio del derecho de acción a través de facilitar el libre acceso a la justicia, con la necesidad de evitar o sancionar el abuso de aquellas premisas mediante la presentación de demandas carentes de razón o, peor aún, hechas de mala fe. Es en este contexto que venimos analizando los dos primeros incisos del artículo 427 del Código Procesal Civil, centrándose nuestra preocupación en que una interpretación excesivamente rígida de aquellos dos supuestos prive a alguna persona de ese manto protector que es el «acceso a la justicia», tanto más que, como hemos visto, la legitimidad para obrar y el interés son elementos a apreciarse en la sentencia y no deben condicionar la demanda, cuya presentación material, insistimos, es la concreción del ejercicio del derecho de acción, sólo posible con un libre acceso a la justicia.

No bastan, en nuestro concepto, para atenuar ese riesgo, que lo es en un medio como el nuestro, los adverbios «evidentemente» y «manifiestamente» contenidos en el texto de los incisos 1 y 2 respectivamente del artículo 427, pues además que toda decisión humana, y la del juez lo es también, importa la expresión siempre subjetiva de un ser humano, pensamos que la inclusión de tales términos grafica mejor que cualquier otra consideración el temor subyacente a que ocurra precisamente lo que estamos te-

8 FIX Zamudio, Héctor, ponencia a las IX Jornadas Iberoamericanas de Derecho Procesal, celebradas en Madrid, España, en junio de 1985, pág. 4. 
miendo: el rechazo apresurado de una demanda recortando, posiblemente de buena fe, el acceso a la justicia, que en un momento como el actual resulta decisivo para lograr o por lo menos mantener la tan necesaria paz social.

Valga también este comentario, aunque reconozcamos el acierto de la concesión de la apelación prevista en el último párrafo del artículo 427 , medida conveniente, sin duda, pero que no cambia lo esencial de la cuestión.

En el propio texto del Código encontramos otra razón que asiste la conveniencia de considerar la legitimidad para obrar y el interés como elementos a ponderarse en la sentencia y no como condicionantes de la tramitación de la demanda. En efecto el artículo IV de su Título Preliminar establece en su primer párrafo que: «El proceso se promueve sólo a iniciativa de parte, la que invocará interés y legitimidad para obrar. No requieren invocarlos el Ministerio Público, el procurador oficioso ni quien defiende intereses difusos». Quien invoca, lo que hace es expresar, hacer presente al juez, que interpone la demanda porque está premunido de ambos elementos y por ello peticiona ante la autoridad judicial. Incluso, la exigencia contenida en el inciso 4 del artículo 425 abunda a favor pues la prueba de la calidad de heredero, cónyuge, etc., acredita in limine la legitimidad, pero juzgamos inconveniente que su no presentación con la demanda, considerando aquella, la prueba, como razón determinante de la legitimidad, origine la paralización del proceso y produzca, en la práctica, una limitación al acceso a la justicia, tanto más que se trata de elementos propios de la evaluación que el juez ha de realizar en la sentencia, y no condicionantes, repetimos, de la admisión y tramitación de la demanda.

Algo más: resulta inevitable apreciar la norma mencionada del primer párrafo del artículo IV del Título Preliminar del Código Procesal Civil, con lo que establece, al respecto, la legislación sustantiva.

El artículo IV del Título Preliminar del Código Civil de 1936 dice: «Para ejercitar o contestar una acción es necesario tener legítimo interés económico o moral. El interés moral sólo autoriza la acción cuando se refiere directamente al agente o a su familia, salvo disposición expresa de la ley». Disposición similar la encontramos en el artículo VI del Título Preliminar del Código Civil vigente de 1984.

Se trata, como se ve, de ejercitar, el demandante, o contestar, el de- 
mandado; en otras palabras, ambos, el demandante y el demandado, proponen al juez la demanda y la contestación, invocando, sosteniendo que tienen la legitimación y el interés, pero ambos conceptos han de dilucidarse en la sentencia. Al respecto nos enseña León Barandiarán: «De esta suerte se comprende que, salvo excepciones expresas, sin mayores esfuerzos, sólo el titular del derecho, o a lo sumo otras personas determinadas dentro de un ámbito siempre reducido, pueden interponer una acción, por tener la pertinente capacidad de obrar». Interponer, dice, no otro término. Y en la relación con el interés moral: «Solf, refiriéndose al interés moral escribe: el interés moral es un concepto flotante que impregna el Derecho y por ello no puede ser fijado de antemano. La sentencia judicial es la llamada a definir la controversia, a certificar el interés moral del demandante y del demandado, salvo los casos de decisión espontánea del agresor»".

Al interponer la demanda o al contestarla, demandante y demandado, invocan, alegan, la legitimidad y el interés. La sentencia dirá si tienen o no razón. Al decidir el juez, no antes.

Desde el punto de vista sistémico nos reafirmanos en nuestra posición. El artículo IV del Código Procesal Civil y el artículo VI del Código Civil se encuentran en los Títulos Preliminares de ambos cuerpos de leyes e informan, como tales, los principios y textos de los mismos.

Una visión panorámica de la legislación comparada nos sigue convenciendo de esta tesis.

El artículo 1 del Código de Procedimientos Civiles para el Distrito Federal de México, dice: «Sólo puede iniciar un procedimiento judicial o intervenir en él, quien tenga interés en que la autoridad judicial...», no incluyendo el artículo 255 como requisito de la demanda, ni la legitimidad ni el interés. Similar solución se advierte en los artículos 1 y 322 del Código Federal de Procedimientos Civiles del mismo México.

9 León Barandiarán, José, «Comentarios al Código Civil peruano», Revista de Derecho y Ciencias Políticas de la Facultad de Derecho de la Universidad Nacional Mayor de San Marcos, año XII, número II, Lima, MCMXLVIII, págs. 278-280. 
El segundo párrafo del artículo 51 del Código Procesal Civil y Mercantil de Guatemala, de 1964, establece que: «Para interponer una demanda o contrademanda es necesario tener interés en la misma», sin que su artículo 106 haga referencia a la legitimidad y al interés entre los requisitos de la demanda.

El artículo 330 del Código Procesal Civil y Comercial de la Nación (ley 17454 de 1967) de la Argentina, tampoco incluye dichos conceptos al legislar sobre el contenido de la demanda.

El artículo 3 del Código Procesal Civil de Brasil, de 1973, dice que: «Para proponer o contestar una acción es necesario tener interés y legitimidad», manteniéndose la misma tónica respecto de los requisitos de la demanda en el artículo 282.

Igual situación se advierte en el primer párrafo del artículo 16 del Título Preliminar del Código de Procedimiento Civil de Venezuela de 1986: «Para proponer la demanda el actor debe tener interés jurídico actual»; y artículo 340 en lo que concierne a los requisitos de la demanda que no se refieren expresamente a los conceptos comentados.

El artículo 99 del Código Procesal Civil de la República del Paraguay, de 1988, admite una diversa connotación del término «interés», al establecer que: «El interés del que propone la acción podrá limitarse a la declaración de la existencia o no existencia de una relación jurídica o a la declaración de autenticidad o falsedad de un documento", sin que su artículo 215 incida en la legitimación o en el interés comentados.

Igual situación acontece con el artículo 75 del Código de Procedimiento Civil de Colombia, de 1971, respecto de los requisitos de la demanda, si bien en el inciso 5 del artículo 77 se señala, como anexo de la demanda, «La prueba de la calidad de heredero, cónyuge, curador de bienes, administrador de comunidad o albacea con que actúe el demandante o se cite al demandado», y aunque el inciso 2 del artículo 85 establece que el juez declarará inadmisible la demanda «cuando no se acompañen los anexos ordenados por la ley», los artículos 78 y 79 del mismo Código establecen excepciones a dicha regla.

El Código General del Proceso de Uruguay, de 1989 (ley 15982), 
adopta el sistema generalmente aceptado, pues en el artículo 11.2 estatuye que: «Para proponer o controvertir útilmente las pretensiones, es necesario invocar interés y legitimación en la causa», sin que en la forma y contenido de la demanda (artículo 117) se señale tales conceptos, tratándose pues de invocar, como lo hacen, como hemos visto, nuestros Código Civil y Código Procesal Civil.

Idéntica solución se advierte en el Código Procesal Civil Modelo para Iberoamérica, cuyos artículos 11.2 y 110 mantienen el mismo texto de sus homólogos del Código uruguayo, identidad que no debe sorprender pues los mismos juristas uruguayos, doctores Enrique Véscovi, Adolfo Gelsi y Luis Torello, fueron los autores del citado Código de su país y ponentes del Código Modelo para América Latina.

Sin embargo, ambos cuerpos de leyes confieren al juez amplias facultades al calificar la demanda, al establecer el artículo 24 inciso 1 del Código uruguayo, que el tribunal está facultado: «Para rechazar in limine la demanda cuando fuere manifiestamente improponible, cuando carezca de los requisitos formales exigidos por la ley o cuando se ejercite una pretensión especialmente sujeta a término de caducidad y éste haya vencido», texto idéntico al contenido en el artículo 33 inciso 1 del Código Modelo, regla que se reproduce en el artículo 119.2 del Código uruguayo y en el artículo 112.2 del Código Modelo.

En sucesivas ocasiones, especialmente en las Jornadas del Instituto Iberoamericano de Derecho Procesal, institución bajo cuyo patrocinio se esbozó, elaboró y aprobó el mencionado Código Modelo, nos hemos pronunciado por el riesgo que entraña la aplicación de tales reglas a partir de un celo excesivamente subjetivo del juez, que puede significar, en la práctica, una traba al acceso a la justicia, como lo hacemos ahora al comentar los artículos 426 y 427 inciso 1 y 2 del nuevo Código Procesal Civil.

El artículo 254 del Código de Procedimiento Civil de Chile, según la edición oficial aprobada por decreto 660 del 30 de abril de 1990, señala el contenido de la demanda, manteniendo los requisitos acostumbrados, sin referencia expresa a la legitimación y al interés del demandante.

En cuanto al Código Procesal Civil de la República de Costa Rica, aprobado por el decreto 7130 de su Asamblea Legislativa y vigente desde 
1990, precisa en su artículo 290 el contenido de la demanda, sin que se incluyan, entre los requisitos, la legitimación y el interés. Independientemente de ello, el artículo 104 establece que «parte legítima», es «aquella que alega tener una determinada relación jurídica con la pretensión procesal», y el artículo 121, teniendo como membrete «La pretensión procesal», dispone que «la persona que pretenda la declaratoria de un derecho a su favor, o la declaración de certeza de una situación jurídica, podrá pedirlo mediante la demanda o la contrademanda».

Es posible pues iniciar el proceso mediante la presentación de una demanda, sin tener que justificar a priori la legitimación o el interés, ambos necesarios, desde luego, para obtener una sentencia favorable, pero que no son condicionantes del ejercicio del derecho de acción.

Tampoco se refiere el artículo 524 de la Ley de Enjuiciamiento Civil de España, a la legitimación y al interés entre los requisitos de la demanda.

El Código de Procedimiento Civil italiano de 1940, vigente desde 1942, precisa en su artículo 100, que: «Para proponer una demanda o para oponerse a la misma, es necesario tener interés en ello».

Estimamos ver en estas normas legislativas, algunas de muy reciente data, una constante respecto a los requisitos de la demanda, que permitan su tramitación, entre ellos los conocidos relativos a los nombres de las partes, la fijación precisa de la materia sub-litis, que no es otra cosa que el objeto del proceso, la fundamentación de hecho y de derecho, etc., con las cuales no cabe disentir. Pero si se advierte bien, ellos son propios de la misma demanda, es decir, del acto jurídico procesal que inicia el proceso. Incluso coincidimos con la necesidad de acompañar los anexos de la demanda a la que se refiere el artículo 425 del Código Procesal Civil, entre ellos la prueba predeterminada de la legitimación contenida en su inciso 4, en aras de una celeridad que tanta falta nos hace. Pero lo que nos preocupa es, insistimos, que, con una aplicación excesivamente formal o subjetiva de los incisos 1 y 2 del artículo 427, se trabe el derecho al acceso a la justicia, que debe ser sagrado, pues su viabilidad no sólo consagra el derecho de accion, derecho humano como hemos visto - y no sólo la demanda sino la justicia-, sino que mantiene el derecho a la jurisdicción como el valioso protector de los derechos de las personas, quienes se sienten así protegidas a priori y hasta cierto punto confiadas en que una autoridad los escuchará y atenderá sus pe- 
didos, no ciertamente para ampararlos todos, pero sí para considerarlos, evaluarlos y expedir la decisión que corresponda. La persona puede ser negada en todas sus pretensiones. Pero al mismo tiempo, debe ser atendida y escuchada en todas ellas. Tal es el ejercicio de la acción, que no admite condicionantes ni limitaciones. La legitimación para obrar y el interés son propios de una sentencia favorable, que requiere para su emisión que aquéllos sean acreditados. Pero no son, no deben ser condicionantes del trámite de una demanda, a la que hemos definido como la expresión material de la acción que afirma y sostiene una pretensión.

Así las cosas y con el ánimo permamente de tratar de equilibrar la necesidad de la reforma procesal con la seguridad que ella debe traer a quienes se proclaman titulares de algún derecho y pretenden que se les reconozca, el artículo 427 del Código Procesal Civil nos merece en definitiva el comentario siguiente:

Los incisos 1 y 2 , atentas las razones expuestas, deben ser interpretados restrictivamente y que, en caso de duda, el juez ha de tramitar la demanda, considerándose la posibilidad de ampliar su texto en tal sentido.

Mantener el inciso 3 por la naturaleza de la caducidad y porque guarda la coherencia con el artículo 2006 del Código Civil.

Mantener asimismo el inciso 4, no obstante que la incompetencia puede deducirse como excepción según el inciso 1 del artículo 446, y además la competencia es un presupuesto procesal, esto es, una de las condiciones para la validez del proceso y siempre constituye una garantía la regla contenida en el último párrafo del comentado artículo 427.

En cuanto a sus tres últimos incisos, no obstante la indudable buena fe del legislador, inspirado en la necesidad de la celeridad procesal y en la conveniencia de evitar o sancionar demandas maliciosas o absurdas, con lo cual convenimos, acusan cierta vaguedad y eventualmente puede prevalecer en su aplicación un exceso de subjetivismo, por lo que, consideramos, que al igual que en el caso de los dos primeros incisos, los supuestos que ellos prevén deben ser apreciados con suma cautela y ponderación, a efecto de que si hubiere duda se prefiera tramitar la demanda, asegurándose el acceso a la justicia, pues ello no implica en modo alguno que necesariamente ha de hacerse lugar a la demanda al expedirse la correspondiente sentencia. 
Pensamos que el mejor homenaje que puede hacerse al esfuerzo que supone la elaboración de un Código Procesal Civil como el que nos rige hace tan poco tiempo, es señalar y comentar los aspectos o temas en los que puede ser perfeccionado, reconociéndose así los aciertos que puede exhibir.

Creemos que todo comentario que se haga sobre el nuevo Código Procesal Civil debe tener siempre como inspiración el cuarto de los mandamientos del inolvidable Eduardo J. Couture. 